\title{
Clinico-Biochemical Profile of Children with Congenital Anomalies of the Kidney and Urinary Tract: A Cross-Sectional Study
}

\author{
Veerabhadra Radhakrishna $^{a}$ Sambandan Kumaravel ${ }^{a}$ \\ P.S. Priyamvada ${ }^{b}$ Nandeesha Hanumanthappa ${ }^{c}$ Bibekanand Jindal ${ }^{a}$ \\ Krishnakumar Govindarajan ${ }^{a}$ Bikash Kumar Naredi ${ }^{a}$ Ashok Rijhwani ${ }^{a}$ \\ a Department of Pediatric Surgery, Jawaharlal Institute of Postgraduate Medical Education and \\ Research (JIPMER), Pondicherry, India; ${ }^{b}$ Department of Nephrology, JIPMER, Pondicherry, India; \\ 'Department of Biochemistry, JIPMER, Pondicherry, India
}

\section{Keywords}

Beta-2-microglobulin - Congenital anomalies of the kidney and urinary tract $\cdot$ Estimated glomerular filtration rate .

Hypertension · Plasma renin activity

\begin{abstract}
Background: Congenital anomalies of the kidney and urinary tract (CAKUT) are a group of disorders responsible for the majority of pediatric end-stage renal disease cases. There are only a few studies on CAKUT. Objectives: A study was conducted to determine the clinical and biochemical profile of children with CAKUT and to estimate the prevalence and the factors associated with hypertension in CAKUT. Methods: A cross-sectional study was conducted in a tertiary center for 18 months from March 2014 to August 2015. Demographic data were recorded, and clinical examination including blood pressure measurement was performed. Various biochemical parameters including plasma renin activity (PRA), urinary beta-2-microglobulin (B2M),
\end{abstract}

and microalbuminuria were evaluated. Results: A total of 81 patients with CAKUT were studied. Twenty-two (27\%) patients were underweight, 4 (5\%) patients were stunted, and $26(32 \%)$ were both underweight and stunted. Children with bilateral disease had a higher incidence of underweight (21/44 vs. $8 / 37 ; p=0.04 ; 95 \% \mathrm{Cl}$; Fisher exact test), and both underweight and stunted (25/44 vs. $10 / 37 ; p=$ 0.006 ; $95 \% \mathrm{Cl}$; Fisher exact test) compared to children with unilateral disease. Hypertension was found in $27 \%$ cases. No association was found between hypertension and PRA, $\mathrm{BM} 2$, or microalbuminuria in our study. PRA was inversely proportional to the estimated glomerular filtration rate (eGFR) (Pearson test; $95 \% \mathrm{Cl} ; p=0.006)$. Conclusions: Bilateral disease in CAKUT was significantly associated with poor somatic growth. PRA was inversely proportional to eGFR. The prevalence of hypertension was higher in children with CAKUT than in normal children and is possibly multifactorial as it was not associated with elevated PRA, B2M, or microalbuminuria.

(c) 2018 S. Karger AG, Basel

\section{KARGER}

(c) 2018 S. Karger AG, Basel

E-Mail karger@karger.com

www.karger.com/kdd
S. Kumaravel

Department of Pediatric Surgery JIPMER

Pondicherry, 605006 (India)

E-Mail drskvel@gmail.com 


\section{Introduction}

Congenital anomalies of the kidney and urinary tract (CAKUT) are a group of congenital diseases which include renal anomalies, pelviureteric anomalies, vesicoureteric junction anomalies, and anomalies of the bladder and urethra [1]. CAKUT has an incidence of 1 in 500 live births and is responsible for recurrent urinary tract infection (UTI), hypertension, and chronic kidney disease (CKD), and so on. CAKUT is the leading cause of endstage renal disease (ESRD) in children [2]. Although CAKUT is associated with significant morbidity in children, there are only a few studies in the literature. This influenced us to conduct this study.

The primary objective of the study was to determine the clinical and biochemical profile of children with CAKUT. The secondary objectives were to estimate the prevalence of hypertension in CAKUT and to determine the factors associated with hypertension in CAKUT in a tertiary hospital.

\section{Material and Methods}

A cross-sectional study was conducted in a tertiary center over a period of 18 months from March 2014 to August 2015. The study was started after approval from our Institutional Ethics Committee on Human Studies and was performed in accordance with the Declaration of Helsinki. All neonates and children below 13 years of age were included in the study. Patients with acquired renal abnormalities (e.g., glomerulonephritis, genitourinary tuberculosis, etc.), children with acquired causes of hypertension, such as renovascular hypertension, renal parenchymal infections causing hypertension, and neoplastic causes of hypertension, as well as children with nonrenal causes of hypertension were excluded from the study.

The sample size was calculated with a $95 \%$ confidence interval (CI) with a prevalence of CAKUT of 5\% and an alpha error of 5\%. Informed consent was obtained from the parents of all participants included in the study. The relevant history was taken, clinical examination was done, and findings were noted in a predesigned proforma. Length/height and weight were compared with the Indian Academy of Pediatrics reference charts [3]. A child was considered underweight when weight was $\leq 3 \mathrm{rd}$ percentile, considered stunted when length/height was $\leq 3$ rd percentile, and underweight and stunted when weight and length/height were $\leq 3 \mathrm{rd}$ percentile. Blood pressure was recorded using a noninvasive blood pressure monitor. A systolic/diastolic/mean arterial pressure of more than or equal to the 95th percentile for sex, age, and height for at least three readings was considered as hypertension [4].

Blood samples were collected by sterile puncture of a peripheral vein. Plasma renin activity (PRA) was estimated using an enzyme-linked immune sorbent assay (ELISA) kit by Vishat Diagnostics Pvt. Ltd. PRA values were compared with the reference value corresponding to the age. Normal PRA values were: $<1$ year $=4-8 \mathrm{ng} / \mathrm{mL} / \mathrm{h} ; 1-3$ years $=1-9 \mathrm{ng} / \mathrm{mL} / \mathrm{h} ; 3-6$ years $=1-5 \mathrm{ng} /$ $\mathrm{mL} / \mathrm{h} ; 6-13$ years $=1.4-2.6 \mathrm{ng} / \mathrm{mL} / \mathrm{h})[5]$.

Urinary beta-2-microglobulin (B2M) was estimated using the ELISA kit by Immunoshop. A urine B2M level of up to $0.3 \mu \mathrm{g} / \mathrm{mL}$ was considered as normal and elevated if the value was more than $0.3 \mu \mathrm{g} / \mathrm{mL}$. Microalbuminuria was estimated using the ELISA kit by Tulip Diagnostics. A urine microalbumin level $>30 \mathrm{mg} / \mathrm{L}$ was considered positive. Creatinine in serum and urine was measured by Jaffe's method traceable to isotope dilution mass spectrometry (IDMS). Urinary protein was measured by the Biuret method. The estimated glomerular filtration rate (eGFR) was calculated by the Modified Schwartz equation: GFR $\left(\mathrm{mL} / \mathrm{min} / 1.73 \mathrm{~m}^{2}\right)=(0.41 \times$ height in $\mathrm{cm}$ )/creatinine in $\mathrm{mg} / \mathrm{dL}$ as defined by NKDEP-NIDDK [6]. The normal range of GFR was defined according to age after Holliday and Barratt [7].

\section{Statistical Methods}

The various demographical parameters and clinical features of children with CAKUT were expressed in percentage. The biochemical parameters were expressed as mean \pm SD. As the biochemical parameters did not follow a normal distribution, they were further expressed as median with range. The association between hypertension and the other factors were analyzed by the Fisher exact test. Two groups were considered significantly different when the $p$ value was $<0.05$ with $95 \%$ CI.

\section{Results}

A total of 81 patients with the diagnosis of CAKUT were studied, and $41 \%$ of the study group were aged $1-5$ years and had been followed up since infancy $(28 \%)$. The youngest patients in this study were 4 days old $(n=3)$, while the oldest patient was a 12-year-old girl. Out of the 81 patients enrolled in the study, $70(86 \%)$ patients were males, while the remaining $11(14 \%)$ patients were females (Table 1).

\section{Types of CAKUT}

Thirty-two (40\%) patients had pelviureteric junction obstruction (PUJO), 26 (32\%) patients had posterior urethral valves (PUV), 15 (19\%) had vesicoureteric reflux (VUR), 5 (6\%) had a multicystic dysplastic kidney (MCDK), 2 (3\%) had ureterocele, and 1 (1\%) had vesicoureteric junction obstruction (VUJO). Of the 11 female patients of the study group, $6(55 \%)$ had PUJO and 5 (46\%) had VUR (Table 1).

Eleven (14\%) out of 81 patients had extrarenal anomalies associated with CAKUT. Four (5\%) patients had anorectal malformation (ARM), 2 (3\%) had undescended testis, and 1 each had a cleft lip and palate, congenital dislocation of the hip, congenital hypothyroidism, congenital talipes equinovarus (CTEV), and congenital glaucoma. 
Table 1. Clinico-biochemical profile of CAKUT

\begin{tabular}{ll}
\hline Variables & $n(\%)$ \\
\hline Age group & \\
Neonates & $10(12)$ \\
Infants & $23(28)$ \\
$1-5$ years & $33(41)$ \\
$\geq 6$ years & $15(19)$ \\
\hline Gender & \\
Males & $70(86)$ \\
Females & $11(14)$ \\
\hline Types of CAKUT & \\
PUV & $26(32)$ \\
VUR & $15(19)$ \\
PUJO & $32(40)$ \\
MCDK & $5(6)$ \\
Ureterocele & $2(3)$ \\
VUJO & $1(1)$ \\
\hline
\end{tabular}

\begin{tabular}{lc}
\hline Clinical presentation & \\
Asymptomatic & $26(32)$ \\
Urinary tract infection & $18(22)$ \\
Urinary dribbling & $18(22)$ \\
Abdominal pain & $15(19)$ \\
Palpable abdominal mass & $7(9)$ \\
Urosepsis & $6(7)$ \\
Hematuria & $3(4)$ \\
Urinary ascites & $1(1)$ \\
Hypertension & $22(27)$ \\
Underweight & $22(27)$ \\
Stunted & $4(5)$ \\
Underweight and stunted & $26(32)$ \\
\hline Biochemical parameters & \\
Raised PRA & $52(64)$ \\
Raised B2M & $52(64)$ \\
Microalbuminuria & $36(44)$ \\
Reduced GFR & $60(74)$ \\
Raised urine PCR & $53(65)$
\end{tabular}

PUV, posterior urethral valves; VUR, vesicoureteric reflux; PUJO, pelviureteric junction obstruction; MCDK, multicystic dysplastic kidney; VUJO, vesicoureteric junction obstruction; PRA, plasma renin activity; B2M, beta-2-microglobulin; GFR, glomerular filtration rate; PCR, protein-creatinine ratio.

\section{Antenatal Diagnosis}

Out of 81 patients enrolled in the study, 75 (93\%) patients had undergone at least one ultrasonogram (USG) in either second or third trimester, and $23(31 \%)$ of these patients had normal USG findings. Fifty (62\%) patients were diagnosed with and $3(4 \%)$ were suspected to have a renal anomaly antenatally. Out of the 81 study participants, 60 (74\%) underwent second-trimester SG. Among these 60 patients, 42 (70\%) had normal USG findings. Six (14\%) out of the 42 patients with normal second trimester USG findings did not undergo a USG in the third trimester, while $14(33 \%)$ continued to have normal USG findings, $20(48 \%)$ patients were diagnosed with a renal anomaly, and 2 had oligohydramnios raising a suspicion of a renal anomaly. Twenty-two (52\%) of the 42 patients with normal second-trimester USG findings were detected to have new renal anomalies in a third-trimester scan despite a normal second-trimester scan. Antenatally, 31 (41\%) had bilateral disease, 11 (15\%) left-sided disease, and 7 (9\%) patients had right-sided disease.

\section{Clinical Presentation}

Thirty-eight (47\%) children presented in infancy, while $23(28 \%)$ patients presented after 1 year of age. Twenty-six (32\%) were asymptomatic, while 18 (22\%) patients presented with UTI, 18 (22\%) had dribbling, 15 (19\%) had abdominal pain, 7 (9\%) a palpable abdominal mass, $6(7 \%)$ urosepsis, 3 (4\%) hematuria, and 1 (1\%) urinary ascites. Twenty-two (27\%) patients were found to have hypertension. Twenty-six (32\%) were stunted and underweight, while $22(27 \%)$ patients were underweight, and $4(5 \%)$ patients were stunted (Table 1$)$.

\section{Laboratory Investigations}

Fifty-two (64\%) patients had raised urinary B2M in their urine (normal: $\leq 0.3 \mu \mathrm{g} / \mathrm{mL}$ ). The mean B2M in the study group was $2.6 \pm 5.1 \mu \mathrm{g} / \mathrm{mL}$. The median B2M was $0.6 \mu \mathrm{g} / \mathrm{mL}$ (range: $0.05-35.2 \mu \mathrm{g} / \mathrm{mL}$ ). Fifty-two (64\%) patients had raised PRA, with the age group of 6- to 13-yearold children having the highest incidence (77\%). The mean PRA in the study population was $18.8 \pm 30.6 \mathrm{ng} /$ $\mathrm{mL}$. The median PRA value was $9.4 \mathrm{ng} / \mathrm{mL}$ (range: $0.5-$ $200.3 \mathrm{ng} / \mathrm{mL}$ ). Thirty-six (44\%) patients had microalbuminuria (normal: $\leq 30 \mathrm{mg} / \mathrm{L}$ ). Mean microalbuminuria in the study group was $58.7 \pm 67 \mathrm{mg} / \mathrm{L}$ (SD). Median microalbuminuria was $27 \mathrm{mg} / \mathrm{L}$ (range: 1-243 mg/L). Twentyeight $(35 \%)$ patients had a normal protein-creatinine ratio ( $\leq 0.2), 53$ (66\%) had raised PCR, and 9 patients had a nephrotic range of PCR. The mean PCR in the study population was $1.4 \pm 2.2$. The median PCR was 6.6 (range: 0.001-13.2). Sixty (74\%) patients had a reduced eGFR. The mean GFR was $47.4 \pm 17.6 \mathrm{~mL} / \mathrm{min} / 1.73 \mathrm{~m}^{2}$. The median GFR was $47.4 \mathrm{~mL} / \mathrm{min} / 1.73 \mathrm{~m}^{2}$ (range: $4-90.9 \mathrm{~mL} /$ $\mathrm{min} / 1.73 \mathrm{~m}^{2}$ ) (Table 1 ).

\section{Unilateral versus Bilateral Disease}

Children with a bilateral disease were significantly more underweight (21/44 vs. $8 / 37$; $p=0.04$; 95\% CI; Fish- 
Table 2. Unilateral versus bilateral disease

\begin{tabular}{lccl}
\hline Parameters & $\begin{array}{l}\text { Unilateral } \\
\text { disease } \\
(n=37)\end{array}$ & $\begin{array}{l}\text { Bilateral } \\
\text { disease } \\
(n=44)\end{array}$ & $\begin{array}{l}p \text { value } \\
\text { (Fisher } \\
\text { exact test) }\end{array}$ \\
\hline Underweight & $8(22)$ & $21(48)$ & 0.04 \\
Stunted & $2(5)$ & $2(5)$ & 1 \\
Underweight and stunted & $10(27)$ & $25(57)$ & 0.006 \\
Hypertension & $11(30)$ & $11(25)$ & 0.8 \\
Raised PRA & $23(62)$ & $29(66)$ & 0.6 \\
Raised B2M & $25(68)$ & $27(61)$ & 0.6 \\
Microalbuminuria & $16(43)$ & $20(46)$ & 1 \\
Raised PCR & $21(57)$ & $32(73)$ & 0.2 \\
Reduced eGFR & $28(76)$ & $32(73)$ & 0.8 \\
\hline
\end{tabular}

Values in parentheses are percentages. PRA, plasma renin activity; B2M, beta-2-microglobulin; PCR, protein-creatinine ratio; eGFR, estimated glomerular filtration rate.

Table 3. Hypertension versus nonhypertension group

\begin{tabular}{lcll}
\hline Parameters & $\begin{array}{l}\text { Hypertension } \\
(n=22)\end{array}$ & $\begin{array}{l}\text { Non- } \\
\text { hypertension } \\
(n=59)\end{array}$ & $\begin{array}{l}p \text { value } \\
\text { (Fisher } \\
\text { exact test) }\end{array}$ \\
\hline $\begin{array}{l}\text { Underweight } \\
\begin{array}{l}\text { Stunted } \\
\text { Underweight and }\end{array}\end{array}$ & $8(36)$ & $26(44)$ & 0.6 \\
$\quad$ stunted & $6(27)$ & $2(3)$ & 0.3 \\
$\begin{array}{l}\text { Raised PRA } \\
\text { Raised B2M }\end{array}$ & $14(64)$ & $38(64)$ & 0.4 \\
$\begin{array}{l}\text { Microalbuminuria } \\
\text { Raised PCR }\end{array}$ & $9(41)$ & $37(63)$ & 0.8 \\
Reduced eGFR & $13(59)$ & $40(68)$ & 0.8 \\
\hline
\end{tabular}

Values in parentheses are percentages. PRA, plasma renin activity; B2M, beta-2-microglobulin; PCR, protein-creatinine ratio; eGFR, estimated glomerular filtration rate. A logistic regression analysis which included all these parameters yielded an $R^{2}$ of 0.047 (Cox and Snell) with a $p$ value of 0.9 which was not significant.

er exact test) and both underweight and stunted (25/44 vs. $10 / 37 ; p=0.006 ; 95 \%$ CI; Fisher exact test) compared to children with unilateral disease. Median PRA and B2M were found to be higher in bilateral disease, but no statistical significance was found (Table 2).

\section{Hypertension versus Nonhypertension Group}

In the hypertension group, there were more patients with reduced GFR compared to the nonhypertension group, but it was statistically not significant. The hyper- tension group had higher median PRA and B2M compared to the nonhypertension group, but it did not reach statistical significance (Table 3). A logistic regression analysis which included all these parameters yielded an $R^{2}$ of 0.047 (Cox and Snell) and a $p$ value of 0.9 which was not significant.

\section{The Relationship of PRA, B2M, and}

Microalbuminuria with eGFR

PRA was inversely proportional to GFR with a correlation coefficient of -0.3 ( $p=0.006$, Pearson test of correlation), whereas $\mathrm{B} 2 \mathrm{M}$ and microalbuminuria were not found to correlate with GFR ( $p=0.8$ and 0.6 , respectively). PRA was directly proportional to B2M and microalbuminuria, but it was statistically not significant.

\section{Discussion}

CAKUT is a group of congenital diseases which include renal anomalies (renal aplasia and MCDK), pelviureteric anomalies (a duplex collecting system, PUJO, and megaureter), vesicoureteric junction anomalies (VUJO, VUR, and ureterocele), ectopic ureteric orifice, anomalies of the bladder, and anomalies of the urethra (PUV, anterior urethral valves, duplication of urethra, and urethral hypoplasia) [1].

CAKUT is the most common cause of ESRD in children comprising $50 \%$ of cases [2]. CAKUT also accounts for $7 \%$ of adult ESRD worldwide [8]. CAKUT is seen in 1 in 500 newborns [2] and in $0.3-1.6$ per 1,000 live born and stillborn neonates [9]. A study by Bondagji [10] found an incidence of 3.26 per 1,000 live births. A study by Zhang et al. [11] showed an incidence of $1.67 \%$ in the pediatric population. The male-to-female ratio in our study was 6.4:1 which was higher than in the studies by Bondagji [10] (2.13:1) and Zhang et al. [11] (4.26:1). Females had either PUJO or VUR in our study.

In our study, there were $13.6 \%$ of CAKUT patients with nonrenal anomalies, the most common being ARM (36.4\%). Bondagji [10] found associated nonrenal anomalies in $26.2 \%$ of cases and Soliman et al. [8] in $31.8 \%$ of cases. The incidence of the different types of CAKUT in the current study and other studies is shown in Table 3. Like in many other studies, PUJO was the most common CAKUT in our study.

Antenatal suspicion or a diagnosis of CAKUT occurred in $65 \%$ of our study population. CAKUT was detected antenatally in $76 \%$ of cases by Melo et al. [2], $66 \%$ of cases by Scott [12], and 37\% of cases by Soliman et al. 
[8]. In our study, we also found that third-trimester USGs were more sensitive in diagnosing CAKUT antenatally. A total of $52 \%$ of patients with normal second-trimester USGs were diagnosed or suspected to have renal anomaly by third-trimester USGs in our study. This shows the importance of third-trimester USGs in detecting renal anomalies missed by the second-trimester USG. Thus, it ensures prompt and timely postnatal intervention. Manegold-Brauer et al. [13] also support our findings which found an increased rate of detection of fetal anomalies by a third-trimester scan. Hence, it is important not to skip fetal USG in the third trimester, which can help us find out renal anomalies requiring early postnatal intervention.

Even with a remarkable antenatal suspicion/diagnosis of $65 \%, 42 \%$ (22/53 children) presented beyond infancy. Among the antenatally undetected children, 38 (47\%) patients presented in infancy, while 23 (28\%) patients presented after 1 year of age. A study by Caiulo et al. [14] on 171 patients with CAKUT who were diagnosed antenatally and had intervention early found that none of the patients required dialysis during a study period of 19 years. Three out of 171 patients had chronic renal failure [14]. This reflects the importance of early diagnosis and management. A total of $32 \%$ of our study group was stunted as well as underweight, while $27 \%$ of patients were underweight and 5\% were stunted. This finding demonstrates that $64 \%$ of CAKUT patients had an affected growth (generally, $36 \%$ of Indian children have an affected growth according to UNICEF [15]). Somatic growth was more significantly affected in patients with bilateral disease than with unilateral disease (underweight: $p=0.04,95 \%$ CI; stunted: $p=0.006,95 \%$ CI; Fisher exact test). CAKUT, even in the absence of CKD, was associated with poor somatic growth, which needs to be further studied and elucidated.

A total of $32 \%$ of patients in our study were asymptomatic at presentation, and $22 \%$ patients presented with UTI. The majority of patients presenting with UTI reflect a lack of awareness among parents, as $42 \%$ of patients presented at or after 1 year of life even though CAKUT was detected antenatally. In our study, $54 \%$ of patients were found to have bilateral disease, while $46 \%$ had unilateral disease with the left side being more commonly involved (30\%). Melo et al. [2] found that $65 \%$ of their study population had bilateral disease and $35 \%$ had unilateral disease, with the left side being predominant (22\%).

Hypertension is associated with increased morbidity and mortality in children. It can lead to ESRD, damaging

Congenital Anomalies of the Kidney and

Urinary Tract already compromised kidneys. On the other hand, renal disorders constitute approximately $75 \%$ of secondary hypertension cases in childhood [16-18]. The risk of hypertension is directly related to the type of underlying disease rather than to the amount of renal insufficiency. Renal hypoplasia/dysplasia and uropathies cause less blood pressure problems compared to glomerulopathy or polycystic kidney disease [16-18]. The possible factors leading to hypertension in CAKUT are abnormal natriuresis, sodium and water retention, the renin-angiotensin-aldosterone system (RAAS), activation of the sympathetic nervous system, endothelial factors, hyperuricemia, and oxidative stress [16-18].

A total of $27 \%$ of our study population was diagnosed with hypertension. This is higher than the prevalence of hypertension in the general pediatric population which varies from 1 to $3 \%[16,17]$. In a study by Sanna-Cherchi et al. [18], the incidence of hypertension in CAKUT was $8 \%$. In our study, the hypertension group was found to have a higher median PRA and B2M, as well as reduced GFR, compared to the nonhypertension group, but it did not reach statistical significance, suggesting that hypertension may be multifactorial and not depending solely on plasma renin. In our study, we had similar findings to other studies like the one by Savage et al. [19] who found an increase in PRA and blood pressure after 5 years of follow-up, but there was no association between raised PRA and hypertension. A logistic regression analysis showed no significant association between these parameters and hypertension. Our study reconfirms the fact that hypertension is higher in CAKUT and it re-emphasizes a diligent blood pressure recording for all CAKUT cases even if they are asymptomatic.

A total of $64 \%$ of the study group had elevated PRA levels. The highest incidence was found in the group of patients aged between 6 and 13 years, indicating that PRA possibly increases with prolonged duration of renal damage. PRA was found to be inversely proportional to GFR, which was statistically significant ( $p=0.006$; 95\% CI). Median PRA was found higher in the bilateral disease and hypertension groups than in the unilateral disease and nonhypertension groups, respectively, but no statistical difference was found. Savage et al. [19] studied PRA initially and at the 5 -year follow-up in 85 patients with renal scarring. PRA was significantly increased at the 5-year follow-up, but there was no significant correlation between blood pressure and PRA.

Our study also showed that PRA was inversely proportional to GFR with a correlation coefficient of -0.3 ( $p=$ 
0.006, Pearson test of correlation), whereas B2M and microalbuminuria were not found to correlate with GFR. This suggests that PRA can be used as a marker of renal failure. A total of $64 \%$ of our study population had raised urinary B2M, and $44 \%$ of our study group had microalbuminuria. A study by Seeman et al. [20] found that the occurrence of microalbuminuria was significantly higher in children with primary hypertension (suggesting renal damage) compared to white coat hypertension. In Seeman et al.'s study, the prevalence of microalbuminuria was $20 \%$.

In summary, the most frequent types of CAKUT were PUJO and PUV. CAKUT, even in the absence of CKD, was associated with poor somatic growth. Two-thirds of CAKUT in children were diagnosed antenatally. An ultrasound screening in the third trimester is a must as the second-trimester screening USG can miss up to $52 \%$ of CAKUT. Thus, a third-trimester scan can detect these cases, which can give a clue for early postnatal intervention. It is also important that parents of antenatally diagnosed children are adequately counseled so that there is no delay in postnatal evaluation and intervention, if required. PRA had a good predictive value in detecting renal damage as it was inversely proportional to the eGFR. Our study reconfirms the fact that hypertension is higher in CAKUT, and it re-emphasizes a diligent blood pressure recording for all CAKUT even if they are asymptomatic.

\section{Statement of Ethics}

All procedures performed in studies involving human participants were in accordance with the ethical standards of the institutional and/or national research committee at which the studies were conducted (IRB approval No. JIP/IEC/SC/2014/2/547) and with the 1964 Helsinki declaration and its later amendments or comparable ethical standards. Informed consent was obtained from all individual participants included in the study.

\section{Disclosure Statement}

The authors have no conflicts of interest relevant to this article to disclose.

\section{Author Contributions}

Dr. Veerabhadra Radhakrishna designed the study, contributed substantially in the acquisition of data, and reviewed and revised the manuscript. Dr. S. Kumaravel conceptualized and designed the study, acquired and tabulated the data, analyzed the data, and drafted and critically revised the manuscript. Dr. P.S. Priyamvada conceptualized and designed the study, reviewed and revised the manuscript. Dr. Nandeesha designed the data collection instruments, coordinated and supervised the data collection, and critically reviewed the manuscript. Drs. Ashok Rijhwani, Bibekanand Jindal, Krishnakumar Govindarajan, and Bikash Kumar Naredi contributed substantially in the acquisition of data, and reviewed and revised the manuscript. All authors approved the final manuscript as submitted and agree to be accountable for all aspects of the work in ensuring that questions related to the accuracy or integrity of any part of the work are appropriately investigated and resolved.

\section{References}

1 Ichikawa I, Kuwayama F, Pope JC 4th, Stephens FD, Miyazaki Y. Paradigm shift from classic anatomic theories to contemporary cell biological views of CAKUT. Kidney Int. 2002 Mar;61(3):889-98.

2 Melo BF, Aguiar MB, Bouzada MC, Aguiar RL, Pereira AK, Paixão GM, et al. Early risk factors for neonatal mortality in CAKUT: analysis of 524 affected newborns. Pediatr Nephrol. 2012 Jun;27(6):965-72.

3 Khadilkar VV, Khadilkar AV, Choudhury P, Agarwal KN, Ugra D, Shah NK. IAP growth monitoring guidelines for children from birth to 18 years. Indian Pediatr. 2007 Mar;44(3): 187-97.

4 National High Blood Pressure Education Program Working Group on High Blood Pressure in Children and Adolescents. The fourth report on the diagnosis, evaluation, and treatment of high blood pressure in children and adolescents. Pediatrics. 2004 Aug;114(2 Suppl 4th Report):555-76.
5 Bajpai M, Singh A. Plasma renin activity: an early marker of progressive renal disease in posterior urethral valves. J Indian Assoc Pediatr Surg. 2013 Oct;18(4):143-6.

6 Bedside IDMS-traceable Schwartz GFR Calculator for Children. The National Institute of Diabetes and Digestive and Kidney Diseases, Bethesda. Available from: http://www. niddk.nih.gov/health-information/healthcommunication-programs/nkdep/labevaluation/gfr-calculators/childrenconventional-unit/Pages/default.aspx. Accessed July 15, 2017.

7 Holliday MA, Barratt TM. Normal Glomerular Filtration Rate. Paediatric nephrology. Baltimore: Williams \&; Wilkins; 1994. p. 1306.

8 Soliman NA, Ali RI, Ghobrial EE, Habib EI, Ziada AM. Pattern of clinical presentation of congenital anomalies of the kidney and urinary tract among infants and children. Nephrology (Carlton). 2015 Jun;20(6):413-8.
9 Rosenblum ND. Malformation of the Kidney: Structural and Functional Consequences. In: Skorecki K, Chertow GM, Marsden PA, Taal MW, Yu AS, editors. Brenner and Rector's The Kidney. 10th ed. Philadelphia: Elsevier; 2016. pp. 2294-307.

10 Bondagji NS. Antenatal diagnosis, prevalence and outcome of congenital anomalies of the kidney and urinary tract in Saudi Arabia. Urol Ann. 2014 Jan;6(1):36-40.

11 Zhang B, Wang H, Sun N, Jia LQ, Shen Y. [Incidence, diagnosis and treatment of children's congenital abnormalities of the kidney and urinary tract detected in ultrasound screening]. Zhonghua Er Ke Za Zhi. 2011 Jul;49(7): 534-8. Chinese.

12 Scott JE. Fetal, perinatal, and infant death with congenital renal anomaly. Arch Dis Child. 2002 Aug;87(2):114-7. 
13 Manegold-Brauer G, Tercanli S, Struben H, Huang D, Kang A. Is a routine ultrasound in the third trimester justified? Additional fetal anomalies diagnosed after two previous unremarkable ultrasound examinations. Ultraschall Med. 2011 Aug;32(4):381-6.

14 Caiulo VA, Caiulo S, Gargasole C, Chiriacò G, Latini G, Cataldi L, et al. Ultrasound mass screening for congenital anomalies of the kidney and urinary tract. Pediatr Nephrol. 2012 Jun;27(6):949-53.
15 Joint malnutrition country dataset, May 2017, UNICEF, WHO and World Bank Group. Available from: https://data.unicef.org/topic/ nutrition/malnutrition. Accessed October 23 , 2017.

16 Flynn JT. Neonatal hypertension: diagnosis and management. Pediatr Nephrol. 2000 Apr; 14(4):332-41.

17 Schaefer F, Wuhl E. Hypertension in chronic kidney disease. In: Flynn JT, Ingelfinger JR, Portman RJ, eds. Pediatric hypertension. 3rd ed. New York, NY: Springer Science+ Business Media; 2013:323-42. https://doi.org/ 10.1007/978-1-62703-490-6_22.
18 Sanna-Cherchi S, Ravani P, Corbani V, Parodi S, Haupt R, Piaggio G, et al. Renal outcome in patients with congenital anomalies of the kidney and urinary tract. Kidney Int. 2009 Sep;76(5):528-33.

19 Savage JM, Koh CT, Shah V, Barratt TM, Dillon MJ. Five year prospective study of plasma renin activity and blood pressure in patients with longstanding reflux nephropathy. Arch Dis Child. 1987 Jul;62(7):678-82.

20 Seeman T, Pohl M, Palyzova D, John U. Microalbuminuria in children with primary and white-coat hypertension. Pediatr Nephrol. 2012 Mar;27(3):461-7. 